\title{
THE SPACE OF HARMONIC TWO-SPHERES IN THE UNIT FOUR-SPHERE
}

\author{
JOHN BOLTON AND LYNDON M. WOODWARD
}

(Received May 26, 2004, revised April 13, 2005)

\begin{abstract}
A harmonic map of the Riemann sphere into the unit 4-dimensional sphere has area $4 \pi d$ for some positive integer $d$, and it is well-known that the space of such maps may be given the structure of a complex algebraic variety of dimension $2 d+4$. When $d$ less than or equal to 2, the subspace consisting of those maps which are linearly full is empty. We use the twistor fibration from complex projective 3-space to the 4-sphere to show that, if $d$ is equal to 3,4 or 5 , this subspace is a complex manifold.
\end{abstract}

1. Introduction. Every harmonic map from the Riemann sphere $S^{2}$ into the unit 4sphere $S^{4}$ has area $4 \pi d$ for some integer $d$. It has been known for some time $[5,6,10]$ that the space $\operatorname{Harm}_{d}\left(S^{4}\right)$ of such maps may be studied in terms of the twistor lifts of the elements to horizontal holomorphic curves of degree $d$ in complex projective 3 -space $C P^{3}$. It follows from this that $\operatorname{Harm}_{d}\left(S^{4}\right)$ may be given the structure of a complex algebraic variety, and a detailed study of this space has been carried out in $[11,15,16,17]$, where, in particular, it is shown that the complex dimension is $2 d+4$. This result is in line with a conjecture in [2] that the moduli space of harmonic maps of $S^{2}$ into $S^{2 m}$ of degree $d$ is a complex algebraic variety of dimension $2 d+m^{2}$, a result which is known to be true for $d=m(m+1) / 2[1]$ and $d=m(m+1) / 2+1$ [2], and has also recently been proved [8] for general $d \geq m(m+1) / 2$ in the case $m=3$.

A natural topology to put on $\operatorname{Harm}_{d}\left(S^{4}\right)$ is the compact-open topology, and the relationship between this topology and that coming from the complex algebraic variety structure was broached in [9], where it is essentially shown that these topologies coincide.

We also use the twistorial approach to consider the existence or otherwise of singular points of $\operatorname{Harm}_{d}\left(S^{4}\right)$, and prove the following theorem. The final sentence in the statement of the theorem is well-known.

THEOREM 1.1. For $3 \leq d \leq 5$, the space $\operatorname{Harm}_{d}^{\mathrm{LF}}\left(S^{4}\right)$ of linearly full elements of $\operatorname{Harm}_{d}\left(S^{4}\right)$, equipped with the compact-open topology, is a complex manifold of complex dimension $2 d+4$. For $d \leq 2, \operatorname{Harm}_{d}^{\mathrm{LF}}\left(S^{4}\right)$ is empty.

Similar questions for the space of harmonic maps from $S^{2}$ to $C P^{2}$ have been studied in [7] and [12]. In fact, the components of this space consist of the \pm -holomorphic maps of degree $d$, together with harmonic maps of degree $d$ and energy $4 \pi E$, where $E=3|d|+4+2 r$ for some non-negative integer $r$. It is shown in [12] (see also [13]) that these components are

2000 Mathematics Subject Classification. Primary 58D10; Secondary 53C43.

Key words and phrases. Harmonic maps, 2-sphere, twistor fibration. 
smooth manifolds of dimension $6|d|+4$ in the \pm -holomorphic case and $2 E+8$ in the other cases.

The research for this paper was carried out by the named authors, but the final exposition is the responsibility of the first author, since Lyndon Woodward sadly died before the paper was completed.

2. The twistor fibration. We begin by recalling the twistor fibration $\pi: C P^{3} \rightarrow S^{4}$. Regarding $\boldsymbol{H}^{2}$ as a left quaternionic vector space, this is obtained by composing the Hopf map $\rho: \boldsymbol{C} P^{3} \rightarrow \boldsymbol{H} P^{1}$ given by

$$
\rho\left(\left[z_{1}, z_{2}, z_{3}, z_{4}\right]\right)=\left[z_{1}+z_{2} j, z_{3}+z_{4} j\right],
$$

with the canonical identification of $\boldsymbol{H} P^{1}$ and $S^{4} \subset \boldsymbol{H} \oplus \boldsymbol{R}=\boldsymbol{R}^{5}$ given in the usual way by stereographic projection from $(0,0,0,0,-1)$ onto the equatorial 4-plane $\boldsymbol{H}$ in $\boldsymbol{R}^{5}$ which is included in $\boldsymbol{H} P^{1}$ by $[q] \mapsto[q, 1]$. We recall $[4,5]$ that $\pi$ is a Riemannian submersion when $C P^{3}$ is given the Fubini-Study metric of constant holomorphic sectional curvature 1 .

Now consider the vector space $C[z]_{d}$ of polynomials of degree at most $d$, and let $V$ be the subset of $\left(\boldsymbol{C}[z]_{d}\right)^{4}$ consisting of those quadruples of coprime polynomials with maximum degree $d$ for which the map $z \mapsto\left[f_{1}(z), f_{2}(z), f_{3}(z), f_{4}(z)\right]$ is linearly full in $\boldsymbol{C} P^{3}$. Then $V$ is a projective subset of $\left(\boldsymbol{C}[z]_{d}\right)^{4}$, and we identify its projectivisation $P(V)$ with the space of linearly full holomorphic maps of degree $d$ (and hence area $4 \pi d$ ) from $S^{2}$ to $C P^{3}$ in the usual way via

$$
\left[f_{1}, f_{2}, f_{3}, f_{4}\right] \leftrightarrow z \mapsto\left[f_{1}(z), f_{2}(z), f_{3}(z), f_{4}(z)\right]
$$

Here, and subsequently, we use the complex coordinate on $S^{2}$ defined by stereographic projection from the south pole of $S^{2}$ onto the equatorial plane so that, in the usual sense, we may identify $S^{2}$ with $\boldsymbol{C} \cup\{\infty\}$.

A holomorphic curve $\left[f_{1}, f_{2}, f_{3}, f_{4}\right]$ in $\boldsymbol{C} P^{3}$ is horizontal if it intersects each fibre orthogonally. It is well-known [5] that this holds if and only if

$$
f_{1}^{\prime} f_{2}-f_{1} f_{2}^{\prime}+f_{3}^{\prime} f_{4}-f_{3} f_{4}^{\prime}=0 .
$$

Thus, if we define $\Phi_{d}: V \rightarrow \boldsymbol{C}[z]_{2 d-2}$ by

$$
\Phi_{d}\left(f_{1}, f_{2}, f_{3}, f_{4}\right)=f_{1}^{\prime} f_{2}-f_{1} f_{2}^{\prime}+f_{3}^{\prime} f_{4}-f_{3} f_{4}^{\prime},
$$

then $\Phi_{d}^{-1}\{0\}$ is a projective subset of $V$ and, using (1), $P\left(\Phi_{d}^{-1}\{0\}\right)$ is identified with the space of linearly full horizontal holomorphic maps of degree $d$ from $S^{2}$ to $C P^{3}$.

The set $\operatorname{Harm}_{d}^{L F}\left(S^{4}\right)$ is the union of two disjoint subsets, $\operatorname{Harm}_{d}^{+}\left(S^{4}\right)$ and $\operatorname{Harm}_{d}^{-}\left(S^{4}\right)$, with post-composition by the antipodal map of $S^{4}$ giving a bijection between them. Each element of $\operatorname{Harm}_{d}^{+}\left(S^{4}\right)$ has a unique lift to an element of $P\left(\Phi_{d}^{-1}\{0\}\right)$, so that post-composition by $\pi$ gives a bijective correspondence

$$
\pi_{*}: P\left(\Phi_{d}^{-1}\{0\}\right) \rightarrow \operatorname{Harm}_{d}^{+}\left(S^{4}\right) .
$$

The final statement of our theorem is now clear, since $V$ is empty for $d \leq 2$. 
3. The topologies. We give $\left(\boldsymbol{C}[z]_{d}\right)^{4}$ its natural topology as a vector space, and $P\left(\left(\boldsymbol{C}[z]_{d}\right)^{4}\right)$ the identification topology. Then $V$ is an open subset of $\left(\boldsymbol{C}[z]_{d}\right)^{4}$, and $P(V)$ is an open subset of $P\left(\left(\boldsymbol{C}[z]_{d}\right)^{4}\right)$. Subsets of any of these spaces are then given the induced (subspace) topology.

When $\operatorname{Harm}_{d}^{L F}\left(S^{4}\right)$ is given the compact-open topology, post-composition by the antipodal map of $S^{4}$ is a homeomorphism which interchanges $\operatorname{Harm}_{d}^{+}\left(S^{4}\right)$ and $\operatorname{Harm}_{d}^{-}\left(S^{4}\right)$. In this section we use the methods of [9] to show that $\operatorname{Harm}_{d}^{+}\left(S^{4}\right)$ is homeomorphic to the projective algebraic variety $P\left(\Phi_{d}^{-1}\{0\}\right)$ described in the previous section. Since $S^{2}$ is compact, it follows that the compact-open topology coincides with the topology of uniform convergence (see, for example, p. 6 of [14]). In particular, since this is a metric topology, limit points of subsets are precisely limits of sequences of points of the subset which converge in the ambient space.

LEMma 3.1. $\operatorname{Harm}_{d}^{+}\left(S^{4}\right)$ is a closed subset of $\operatorname{Harm}_{d}^{\mathrm{LF}}\left(S^{4}\right)$, and the map $\pi_{*}$ : $P\left(\Phi_{d}^{-1}\{0\}\right) \rightarrow \operatorname{Harm}_{d}^{+}\left(S^{4}\right)$ is a homeomorphism.

PROOF. We first show that $\pi_{*}$ is continuous. Since

$$
\begin{aligned}
P(V) \times S^{2} & \rightarrow S^{4} \\
\left(\left[f_{1}, \ldots, f_{4}\right], z\right) & \mapsto \pi\left[f_{1}(z), \ldots, f_{4}(z)\right]
\end{aligned}
$$

is continuous, so is its restriction to $P\left(\Phi_{d}^{-1}\{0\}\right) \times S^{2}$. It now follows (see, for example, p. 6 of [14]) that $\pi_{*}$ is continuous.

We now show that $\operatorname{Harm}_{d}^{+}\left(S^{4}\right)$ is a closed subset of $\operatorname{Harm}_{d}^{L F}\left(S^{4}\right)$ and that $\pi_{*}$ is a closed map. To do this it suffices to show that if $\left\{x_{i}\right\}$ is a sequence in $P\left(\Phi_{d}^{-1}\{0\}\right)$ such that $\left\{y_{i}\right\}$, $y_{i}=\pi_{*}\left(x_{i}\right)$ for $i \in N$, is convergent to $y_{0} \in \operatorname{Harm}_{d}^{L F}\left(S^{4}\right)$, say, then $\left\{x_{i}\right\}$ has a subsequence which converges in $P\left(\Phi_{d}^{-1}\{0\}\right)$. Suppose then that, for all $i \in N, x_{i}=\left[f_{i, 1}, \ldots, f_{i, 4}\right]$ where g.c.d. $\left(f_{i, 1}, \ldots, f_{i, 4}\right)=1$. Since $x_{i} \in P\left(\left(\boldsymbol{C}[z]_{d}\right)^{4}\right)$ which is compact, $\left\{x_{i}\right\}$ has a subsequence, which without loss of generality we may also write $\left\{x_{i}\right\}$, which converges to $x_{0}$ in $P\left(\left(\boldsymbol{C}[z]_{d}\right)^{4}\right)$. We will now prove that $x_{0} \in P\left(\Phi_{d}^{-1}\{0\}\right)$, which will complete the proof of the lemma.

Let

$$
x_{0}=\left[f_{1}, \ldots, f_{4}\right]=\left[q g_{1}, \ldots, q g_{4}\right],
$$

where $q=$ g.c.d. $\left(f_{1}, \ldots, f_{4}\right)$. Then

$$
\begin{aligned}
y_{0}(z) & =\left(\lim _{i \rightarrow \infty} y_{i}\right)(z)=\lim _{i \rightarrow \infty}\left(y_{i}(z)\right) \\
& =\lim _{i \rightarrow \infty}\left(\left(\pi_{*}\left(x_{i}\right)\right)(z)\right)=\lim _{i \rightarrow \infty} \pi\left(x_{i}(z)\right) \\
& =\lim _{i \rightarrow \infty} \pi\left[f_{i, 1}(z), \ldots, f_{i, 4}(z)\right] .
\end{aligned}
$$

Now, for fixed $z$, the map $e_{z}:\left(\boldsymbol{C}[z]_{d}\right)^{4} \rightarrow \boldsymbol{C}^{4}$ defined by evaluation at $z$ is continuous. Thus $E_{z}:\left(\boldsymbol{C}[z]_{d}\right)^{4} \backslash e_{z}^{-1}\{0\} \rightarrow \boldsymbol{C}^{4} \backslash\{0\}$, defined by restriction of $e_{z}$, is also continuous. Now let $z$ be a complex number for which $q(z) \neq 0$. We may then assume that $\left\{x_{i}\right\}$ is a sequence in $P\left(\left(\boldsymbol{C}[z]_{d}\right)^{4} \backslash e_{z}^{-1}\{0\}\right)$, for otherwise $x_{0} \in P\left(e_{z}^{-1}\{0\}\right)$, which implies that $q(z)=0$, a 
contradiction. Hence, by continuity of the projectivisation of $E_{z}$, we have

$$
\lim _{i \rightarrow \infty}\left[f_{i, 1}(z), \ldots, f_{i, 4}(z)\right]=\left[f_{1}(z), \ldots, f_{4}(z)\right],
$$

so continuity of $\pi$ shows that

$$
y_{0}(z)=\pi\left[f_{1}(z), \ldots, f_{4}(z)\right]=\pi\left[g_{1}(z), \ldots, g_{4}(z)\right] .
$$

We now complete the proof of the lemma as follows. Since $y_{0}$ and $z \mapsto \pi\left[g_{1}(z), \ldots\right.$, $g_{4}(z)$ ] are smooth maps of $S^{2}$ into $S^{4}$ which agree except possibly at a finite number of points, they are equal. Thus $z \mapsto\left[g_{1}(z), \ldots, g_{4}(z)\right]$ is a holomorphic lift of $y_{0}$ and hence, since $y_{0}$ has induced area $4 \pi d$, the degree of this lift is at least $d$. Thus $x_{0}=\left[f_{1}, \ldots, f_{4}\right]=$ $\left[g_{1}, \ldots, g_{4}\right] \in P\left(\Phi_{d}^{-1}\{0\}\right)$. It follows that $\left\{x_{i}\right\}$ converges in $P\left(\Phi_{d}^{-1}\{0\}\right)$, as required.

4. Proof of the theorem. The method of proof is to show that, for $3 \leq d \leq 5$, the zero polynomial is a regular value of $\Phi_{d}$. It will then follow that $\Phi_{d}^{-1}\{0\}$ is a submanifold of $V$ of dimension $4(d+1)-(2 d-1)=2 d+5$, from which the theorem will follow by projectivising and using Lemma 3.1.

We begin by writing down the derivative $\left.d \Phi_{d}\right|_{\mathbf{f}}$ of $\Phi_{d}$ at a point $\mathbf{f}=\left(f_{1}, \ldots, f_{4}\right)$ of $V$. The unusual indexing on the left hand side is to facilitate the writing down of the derivative.

$$
\left.d \Phi_{d}\right|_{\mathbf{f}}\left(h_{2}, h_{1}, h_{4}, h_{3}\right)=\sum_{p=1}^{4}(-1)^{p}\left(f_{p} h_{p}^{\prime}-f_{p}^{\prime} h_{p}\right) .
$$

In order to simplify the calculations we use the results of [3], where canonical forms are obtained for elements of $P\left(\Phi_{d}^{-1}\{0\}\right)$ for $3 \leq d \leq 5$. Firstly, the standard action of the complexified symplectic group $\operatorname{Sp}(2, \boldsymbol{C})$ on $\boldsymbol{C}^{4}$ induces a natural action on $V$ via $(A \mathbf{f})(z)=$ $A(\mathbf{f}(z))$. It is clear from Section 2 of [3] that $\Phi_{d}$ is constant on the orbits of this action, so the rank of $\left.d \Phi_{d}\right|_{\mathbf{f}}$ is equal to the rank of $\left.d \Phi_{d}\right|_{A \mathbf{f}}$. Secondly, for each positive integer $k$, a Möbius transformation $\mu(z)=(\alpha z+\beta) /(\gamma z+\delta)$ induces a diffeomorphism $\tilde{\mu}: \boldsymbol{C}[z]_{k} \rightarrow \boldsymbol{C}[z]_{k}$ given by

$$
(\tilde{\mu} \mathbf{f})(z)=(\gamma z+\delta)^{k}(\mathbf{f}(\mu(z)) .
$$

This in turn induces a diffeomorphism, also denoted by $\tilde{\mu}$, from $V$ to $V$. It is easily checked that if $\mathbf{f} \in V$, then

$$
\Phi_{d}(\tilde{\mu} \mathbf{f})=(\alpha \delta-\beta \gamma) \tilde{\mu}\left(\Phi_{d} \mathbf{f}\right),
$$

so that the rank of $\left.d \Phi_{d}\right|_{\mathbf{f}}$ is equal to the rank of $\left.d \Phi_{d}\right|_{\tilde{\mu} \mathbf{f}}$.

Now let $\mathbf{f} \in \Phi_{d}^{-1}\{0\}$. For $3 \leq d \leq 5$, it follows from Theorem 4.1, Theorem 5.1 and Proposition 6.1 of [3] that there exists $A \in \operatorname{Sp}(2, \boldsymbol{C})$ and a Möbius transformation $\mu$ such that $\tilde{\mu}(A \mathbf{f})$ is a scalar multiple of one of the following two forms.

Case 1: $\tilde{\mu}(A \mathbf{f})=\left(1+\alpha z, \beta z^{d}, f_{3}(z), f_{4}(z)\right)$, where $\beta \neq 0$ and $f_{3}(z), f_{4}(z) \in \boldsymbol{C}[z]_{d}$.

Case 2: $\quad \tilde{\mu}(A \mathbf{f})=\left(1+\alpha z, z^{4}(1+\beta z), z(1+\gamma z), z^{3}(\mu+\delta z)\right), \beta \mu \neq 0$.

Then the rank of $\left.d \Phi_{d}\right|_{\mathbf{f}}$ is equal to the rank of $\left.d \Phi_{d}\right|_{\tilde{\mu}(A \mathbf{f})}$, so we may show that, for $3 \leq d \leq 5$, 0 is a regular value of $\Phi_{d}$ by displaying for each $\mathbf{f}$ in $\Phi_{d}^{-1}\{0\}$ taking one of the two forms 
above, a $(2 d-1)$-dimensional subspace $W$ of $\left(\boldsymbol{C}[z]_{d}\right)^{4}$ which is complementary to the kernel of $\left.d \Phi_{d}\right|_{\mathbf{f}}$.

For both cases, the following lemma will be useful.

Lemma 4.1. Let $f, g \in \boldsymbol{C}[z]$ be non-zero polynomials and let $z^{r}, z^{s}$ be the highest powers of $z$ dividing $f, g$, respectively. Then, if $r+s \geq 1$, we have

(a) $z^{r+s-1} \mid f g^{\prime}-f^{\prime} g$;

(b) if $r \neq s$ and if $z^{t} \mid f g^{\prime}-f^{\prime} g$ then $t \leq r+s-1$.

The proof follows immediately for $r s \neq 0$ from the fact that if $f=z^{r} \hat{f}, g=z^{s} \hat{g}$ for polynomials $\hat{f}, \hat{g}$ with non-zero constant term, then

$$
f g^{\prime}-f^{\prime} g=z^{r+s-1}\left\{(s-r) \hat{f} \hat{g}+z\left(\hat{f} \hat{g}^{\prime}-\hat{f}^{\prime} \hat{g}\right)\right\} .
$$

The proof when just one of $r, s$ is non-zero is similar but easier.

We first dispose of Case 1 . Let $W$ be the $(2 d-1)$ dimensional vector subspace of $\left(\boldsymbol{C}[z]_{d}\right)^{4}$ consisting of elements of the form $\left(h_{2}, h_{1}, 0,0\right.$, ), with $h_{1}$ a linear combination of $z, \ldots, z^{d}$ and $h_{2}$ a linear combination of $z, \ldots, z^{d-1}$. If such an element is in $\operatorname{ker} d \Phi_{d} \mid \mathbf{f}$, then

$$
f_{1} h_{1}^{\prime}-f_{1}^{\prime} h_{1}-\left(f_{2} h_{2}^{\prime}-f_{2}^{\prime} h_{2}\right)=0 .
$$

Lemma 4.1(a) shows that $z^{d} \mid f_{2} h_{2}^{\prime}-f_{2}^{\prime} h_{2}$, so, by (2), $z^{d} \mid f_{1} h_{1}^{\prime}-f_{1}^{\prime} h_{1}$. If $h_{1} \neq 0$, then Lemma 4.1(b) would give the contradiction $d \leq d-1$. Thus $h_{1}=0$, so that $h_{2}$ is a scalar multiple of $f_{2}$ and hence is also zero. The theorem is now proved in this case.

Case 2 is similar. This time we let $W$ consist of elements of $\left(\boldsymbol{C}[z]_{5}\right)^{4}$ of the form $\left(h_{2}, h_{1}, h_{4}, h_{3}\right)$, with $h_{1}$ a linear combination of $z, \ldots, z^{5}, h_{2}$ a scalar multiple of $z^{5}, h_{3}$ a scalar multiple of $z^{5}$, and $h_{4}$ a linear combination of $z^{4}, z^{5}$. In this case, it follows that $z^{8}\left|f_{2} h_{2}^{\prime}-f_{2}^{\prime} h_{2}, z^{6}\right| f_{4} h_{4}^{\prime}-f_{4}^{\prime} h_{4}$, and $z^{5} \mid f_{3} h_{3}^{\prime}-f_{3}^{\prime} h_{3}$. Thus, if $\left(h_{2}, h_{1}, h_{4}, h_{3}\right) \in W \cap$ $\operatorname{ker} d \Phi_{d} \mid \mathbf{f}$, then $z^{5} \mid f_{1} h_{1}^{\prime}-f_{1}^{\prime} h_{1}$. If $h_{1} \neq 0$, we would have, by Lemma 4.1(b), that $5 \leq 4$. Thus $h_{1}=0$. It now follows that $z^{6} \mid f_{3} h_{3}^{\prime}-f_{3}^{\prime} h_{3}$, and a similar argument shows that $h_{3}=0$. Thus $z^{8} \mid f_{4} h_{4}^{\prime}-f_{4}^{\prime} h_{4}$, which leads us to deduce that $h_{4}=0$. But then $h_{2}$ is a scalar multiple of $f_{2}$, so that $h_{2}=0$. This shows that $W \cap \operatorname{ker} d \Phi_{d} \mid \mathbf{f}=\{0\}$, and the theorem is proved.

\section{REFERENCES}

[ 1 ] J. L. BarbosA, On minimal immersions of $S^{2}$ into $S^{2 m}$, Trans. Amer. Math. Soc. 210 (1975), 75-106.

[2] J. Bolton And L. M. Woodward, The space of harmonic maps of $S^{2}$ into $S^{n}$, Geometry and Global Analysis (Sendai, 1993), 165-173, Tohoku Univ., Sendai, 1993.

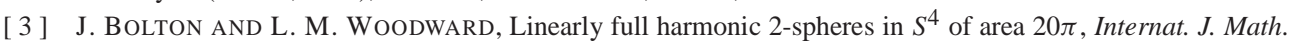
12 (2001), 535-554.

[4] J. Bolton and L. M. Woodward, Higher singularities and the twistor fibration $\pi: C P^{3} \rightarrow S^{4}$, Geom. Dedicata 80 (2000), 231-245.

[ 5 ] R. L. BRYAnt, Conformal and minimal immersions of compact surfaces into the 4-sphere, J. Differential Geom. 17 (1982), 455-473.

[6] S. S. Chern And J. Wolfson, Minimal surfaces by moving frames, Amer. J. Math. 105 (1983), 59-83.

[ 7 ] T. A. CRAWFORD, The space of harmonic maps from the 2-sphere to the complex projective plane, Canad. Math. Bull. 40 (1997), 285-295. 
[ 8 ] L. FernandeZ, The dimension of the space of minimal 2-spheres in $S^{6}$, Preprint.

[9] M. Furuta, M. A. Guest, M. Kotani And Y. Ohnita, On the fundamental group of the space of harmonic 2-spheres in the $n$-sphere, Math. Z. 215 (1994), 503-518.

[10] H. B. LAWSON, JR., Surfaces minimales et la construction de Calabi-Penrose, Séminaire Bourbaki 1983/84, Astérisque 121-122 (1985), 197-211.

[11] B. Loo, The space of harmonic maps of $S^{2}$ into $S^{4}$, Trans. Amer. Math. Soc. 313 (1989), 81-102.

[12] L. Lemaire And J. C. Wood, On the space of harmonic 2-spheres in $\boldsymbol{C} P^{2}$, Internat. J. Math. 7 (1996), 211-225.

[13] L. Lemaire And J. C. Wood, Jacobi fields along harmonic 2-spheres in $\boldsymbol{C} P^{2}$ are integrable, J. London Math. Soc.(2) 66 (2002), 468-486.

[14] E. H. SPANIER, Algebraic Topology, McGraw-Hill Book Co., New York-Toronto, Ont.-London, 1966.

[15] J.-L. VerdieR, Two dimensional $\sigma$-models and harmonic maps from $S^{2}$ to $S^{2 n}$, Lecture Notes in Phys. 180 (1982), 136-141.

[16] J.-L. Verdier, Applications harmoniques de $S^{2}$ dans $S^{4}$, Geometry Today (Rome, 1984), 267-282, Progr. Math. 60, Birkhäuser Boston, Boston, Mass., 1985.

[17] J.-L. Verdier, Applications harmoniques de $S^{2}$ dans $S^{4}$, II, Harmonic mappings, twistors, and $\sigma$-models (Luminy, 1986), 124-147, Adv. Ser. Math. Phys. 4, World Sci. Publishing, Singapore, 1988.

UNIVERSITY OF DURHAM

DEPARTMENT OF MATHEMATICAL SCIENCES

SOUTH ROAD

DURHAM DH1 3LE

GREAT BRITAIN

E-mail address: john.bolton@durham.ac.uk 